\title{
A Compact Cryogenic Pump
}

\author{
Gang Li, ${ }^{\mathrm{a}, \mathrm{b}, \mathrm{c}}$ Shane Caldwell ${ }^{\mathrm{d}, \mathrm{b}}$ \\ ${ }^{a}$ Department of Physics, McGill University, Montréal, Québec H3A 2T8, Canada \\ ${ }^{b}$ Physics Division, Argonne National Laboratory, Argonne, Illinois 60439, USA \\ ${ }^{c}$ Nuclear Science Division, Canadian Nuclear Laboratories, Chalk River, Ontario, K0J 1J0 Canada \\ ${ }^{\mathrm{d}}$ Department of Physics, University of Chicago, Chicago, Illinois 60637, USA
}

\begin{abstract}
A centrifugal cryogenic pump has been designed at Argonne National Laboratory to circulate liquid nitrogen $\left(\mathrm{LN}_{2}\right)$ in a closed circuit allowing the recovery of excess fluid. The pump can circulate $\mathrm{LN}_{2}$ at rates of 2-10 L/min, into a head of 0.5-3 $\mathrm{m}$. Over four years of laboratory use the pump has proven capable of operating continuously for 50-100 days without maintenance.
\end{abstract}

Keywords: Cryogenic pump, Liquid nitrogen, Centrifugal pump

\section{Introduction}

Cryogenic cooling has many applications in modern scientific and engineering research. This type of cooling is commonly achieved by bringing a cryogenic fluid, such as liquid nitrogen $\left(\mathrm{LN}_{2}\right)$, into direct contact with an apparatus by way of a cooling circuit. Often the $\mathrm{LN}_{2}$ is supplied from a closed, pressurized vessel, and in this case the recovery of excess fluid from the circuit is impractical. When a continuous flow is required, the excess fluid is simply discarded. In order to reduce the need for human intervention, it is greatly preferable to recover the excess fluid in a non-pressurized vessel.

$\mathrm{LN}_{2}$ pumps are commercially available from several companies [1,2], but these pumps are rather large and expensive and not well suited to the cooling of small laboratory equipment. A design of a small-scale $\mathrm{LN}_{2}$ pump has been sought for several decades [3-6], but no previous prototype has proven reliable over several weeks of continuous use. In this work we present a compact and affordable design of a centrifugal cryogenic pump, which over the past four years has been used continuously over many intervals of many weeks in our laboratory.

The design of a $\mathrm{LN}_{2}$ pump presents several technical challenges.

(1) Low temperature. Most pumps use flexible material to seal the pump chamber, however flexible materials typically become rigid in liquid nitrogen. Using such materials is not absolutely necessary in a centrifugal pump, but a displacement pump does require a seal to form a trapped volume. This motivates a centrifugal design.

(2) Lubrication. Submerged centrifugal pumps usually utilize the liquid to be pumped as the lubricant for the bearing. But $\mathrm{LN}_{2}$ is a very poor lubricant [7]. An early version of our pump was tested with a ball bearing lubricated by the $\mathrm{LN}_{2}$, and we found that the bearing was badly degraded after one week of continuous operation.

(3) Boiling. Because the $\mathrm{LN}_{2}$ is boiling it cannot be pulled through a circuit by a downstream pump. Instead it must be driven by a positive-pressure device that is submerged in the 
liquid. Even so, as nitrogen vapor fills the pump chamber, the liquid flow is reduced or eliminated.

(4) Cavitation. The boiling liquid is also susceptible to cavitation, in which low-pressure vapor bubbles form and rapidly collapse due to the high strain produced in the fluid by the moving impeller. This cavitation causes a large amount of noise and vibration, pressure pulsation, loss of efficiency, and damage to the pump.

(5) Low specific speed. A pump's performance can be characterized by its specific speed $3.65 n Q / H^{3 / 4}$, where $n$ is the speed of revolution, $Q$ is the flow rate and $H$ is the dynamic head. Generally the applications in small laboratory require a continuous flow of $\mathrm{LN}_{2}$ but not necessarily a high flow rate. Meanwhile the $\mathrm{LN}_{2}$ evaporates when it flows through the circuit, generating a high resistance. These factors tend to limit the pump performance to the low specific speed region, which exacerbates the cavitation problem [8].

In our design, all these obstacles have been overcome. All parts submerged in the $\mathrm{LN}_{2}$ are made of aluminum, stainless steel, or graphite, which are not too much altered by the cryogenic environment. The cavitation problem is solved by (a) using an inducer to increase the pressure in the impeller region, and (b) leaving a strategically placed gap in the pump chamber to allow any gas therein to escape.

\section{Pump design}

The basic concept of the design is to separate the pump housing from the motor by a long supporting tube and a long shaft inside so that the motor will not be frozen by the $\mathrm{LN}_{2}$. Drawings of the pump are shown in Fig. 1(a-c). The motor is DC-driven so that the pump speed can easily be controlled by an adjustable DC power supply. Vibration becomes an important issue when two moving parts are connected by a long shaft. To reduce vibration, two precision ball bearings are used to constrain the top and bottom of the shaft. Every component related to the alignment is designed within $0.1 \mathrm{~mm}$ precision. The lower bearing, which must work in $\mathrm{LN}_{2}$, is a stainless steel bearing lubricated by graphite made by Barden Corporation [9]. The pump shaft and motor shaft were connected by a flexible coupler to provide a tolerance to misalignment, balance the axial force, and absorb vibrations. Under load the coupler was stretched by $0.25 \mathrm{~mm}$, and no axial vibration was measured or observed during operation.

The design of the pump housing, impeller and outlet (Fig. 1(c,d)) is based on a partial emission pump type [10] which is especially suitable for low specific speed. In the partial emission structure only a portion of the liquid flows out through the emission throat oriented tangentially to the housing wall, while the rest of the liquid keeps circulating inside of the housing.

The semi-open impeller has three curved blades fixed on a disk and a central hole to couple with the shaft. The vane shape is approximately an Archimedean spiral. The impeller diameter of $5.0 \mathrm{~cm}$ was chosen in order to generate a head of $2 \mathrm{~m}$ at 40 revolutions per second (rps).

A co-rotating inducer, shown in Fig. 1(e), was installed below the impeller to relieve the strain between the liquid reservoir and the rotating fluid in the impeller region. This raises the local boiling point and reduces cavitation. The inducer has the structure of an Archimedean screw and works as an Archimedean screw pump [11]. 
Nitrogen gas in the pump chamber is allowed to vent via a special gas-release structure, shown in Fig. 1(c). Gas flows away from the impeller region without obstructing the liquid port and without presenting a large conductance to the liquid. This mechanism is based on the structure of the "gas-release plate," which is attached firmly to the pump housing and acts as the lid of the housing. But instead of sealing the pump housing, the plate has a central hole of diameter $20 \mathrm{~mm}$ through which the nitrogen gas can exit the pump housing. A gap of $0.8 \mathrm{~mm}$ is maintained above (between the gas release plate the mounting flange) and below (between the gas release plate and the impeller base) the gas release plate to allow the gas to escape to the liquid reservoir. Whereas in other centrifugal designs the housing is sealed except for the inlet and outlet, this gas release structure alleviates the problems associated with the buildup of $\mathrm{N}_{2}$ gas in the pump housing and circuit. Note that the pumping of the liquid in a centrifugal pump does not mainly rely on the pressure inside the pump housing; rather it is from the movement of the liquid. The liquid prefers to flow through the pump outlet where the speed is maximized; therefore a gas release hole in the center of the pump housing does not noticeably reduce the dynamic head. The semi-open structure of the impeller complies with the gas release mechanism. The flat base of the semiopen impeller (in contrast to the fully open vanes of some other designs), prevents the direct loss of the liquid lifted by the inducer.

The assembled pump is about $90 \mathrm{~cm}$ height and weights a few $\mathrm{kg}$. The majority parts of the pump (except for the motor and bearings) are originally designed. The design specifications of essential parts are listed in Table 1.

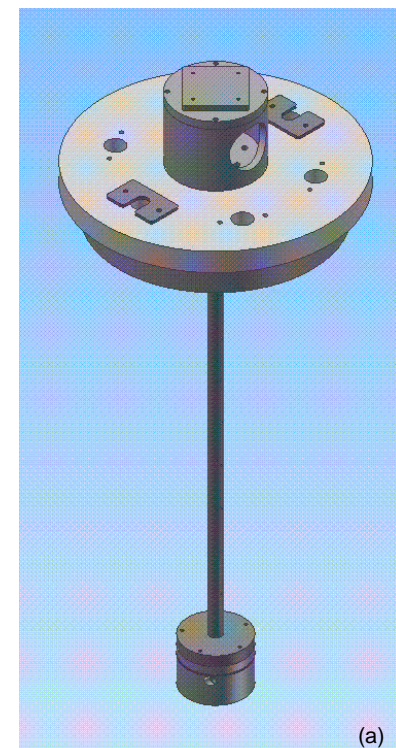

(a)
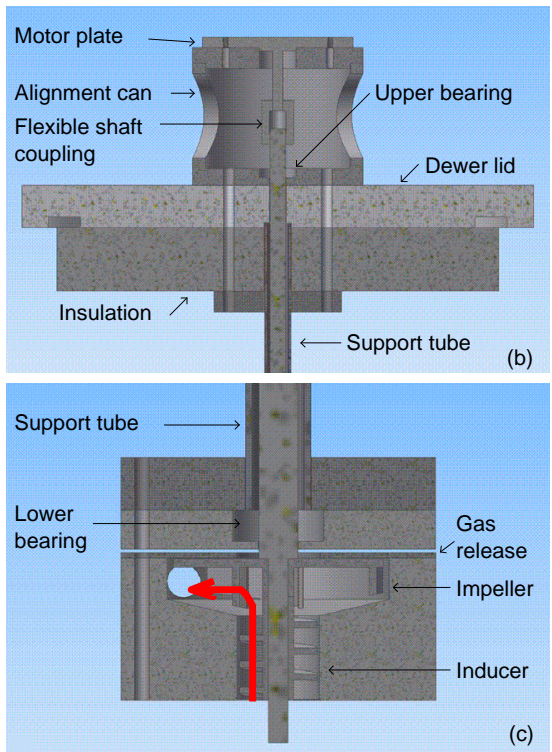

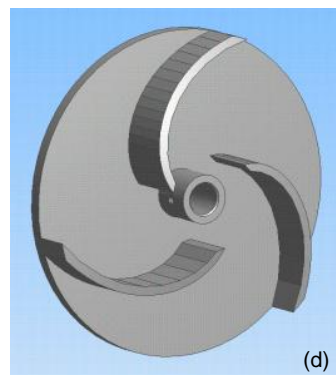

(d)
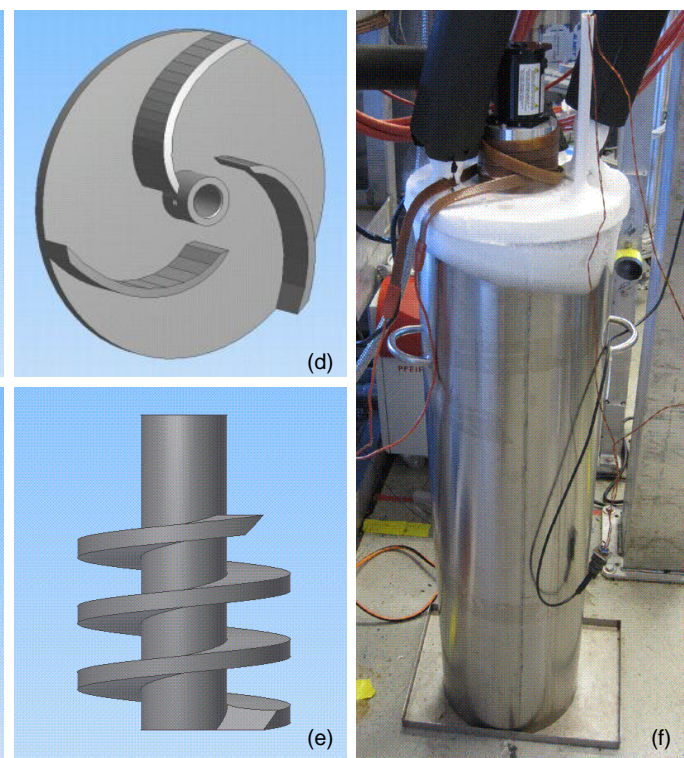

Fig. 1. (a) Assembled centrifugal pump; (b) Breakout of the upper portion, including the motor, flexible shaft coupler, upper bearing, Dewar lib, and shaft; (c) Breakout of the lower (submerged) portion with arrow indicating the direction of flow on the left side. (The flow on the right side is not shown but is cylindrically symmetric about the shaft.) (d) The pump impeller, responsible for driving the liquid through the circuit; (e) The pump inducer, responsible for reducing cavitation; (f) Photo of an operating pump. 
Table 1. Major design specifications.

\begin{tabular}{|c|l|l|}
\hline \multirow{4}{*}{ Item } & Properties & values \\
\hline \multirow{4}{*}{ Shaft } & diameter & $9.5 \mathrm{~mm}$ \\
\cline { 2 - 3 } & length & $65.3 \mathrm{~cm}$ \\
\cline { 2 - 3 } & material & stainless steel \\
\hline \multirow{4}{*}{ Impeller } & diameter & $50.8 \mathrm{~mm}$ \\
\cline { 2 - 3 } & vane height (inner edge) & $10.0 \mathrm{~mm}$ \\
\cline { 2 - 3 } & vane height (outer edge) & $6.0 \mathrm{~mm}$ \\
\cline { 2 - 3 } Inducer & material & aluminium \\
\hline \multirow{3}{*}{$\begin{array}{c}\text { typical } \\
\text { operation properties }\end{array}$} & diameter & $18.0 \mathrm{~mm}$ \\
\cline { 2 - 3 } & screw pitch & $5.8 \mathrm{~mm}$ \\
\cline { 2 - 3 } & material & aluminium \\
\cline { 2 - 3 } & temperature & $77 \mathrm{~K}$ \\
\cline { 2 - 3 } & shaft rotation speed & $20-60 \mathrm{rps}$ \\
\cline { 2 - 3 } & head & $0.5-2 \mathrm{~m}$ \\
\hline & flow rate & $2-10 \mathrm{~L} / \mathrm{min}$ \\
\hline
\end{tabular}

\section{Pump performance}

Operating at $40 \mathrm{rps}$, the pump delivers about $2 \mathrm{~L} / \mathrm{min}$ of $\mathrm{LN}_{2}$ into a head of $2 \mathrm{~m}$. The normal operation frequency ranges from $20 \mathrm{rps}$ to $60 \mathrm{rps}$, offering a wide range of flow rate and pressure. Measurements of flow rate at various pumping heads and operation frequencies are presented in Fig. 2. Over the past four years, four of these pumps have been built and used in various critical applications [12-14] in our laboratory. The flow rates, resistances to flow, and rates of heat are different across our applications, and the pumps have produced good cooling in every case. They have been operated for continuous periods ranging from 1 week to several months. In order to achieve continuous operation, the reservoir level is measured by thermal diodes which open and close a valve to an external supply Dewar, and the external supply is replaced as needed. It has been found necessary to warm the alignment structure below the motor with heating tape to prevent the formation of ice within the structure, and the flexible coupling has been the leading cause of failure. Over many weeks a large bulk of ice builds up around the supply and return lines at their interfaces with the reservoir. Future improvements to the insulation of the Dewar lid and alignment structure from the reservoir and supply lines may be considered. 


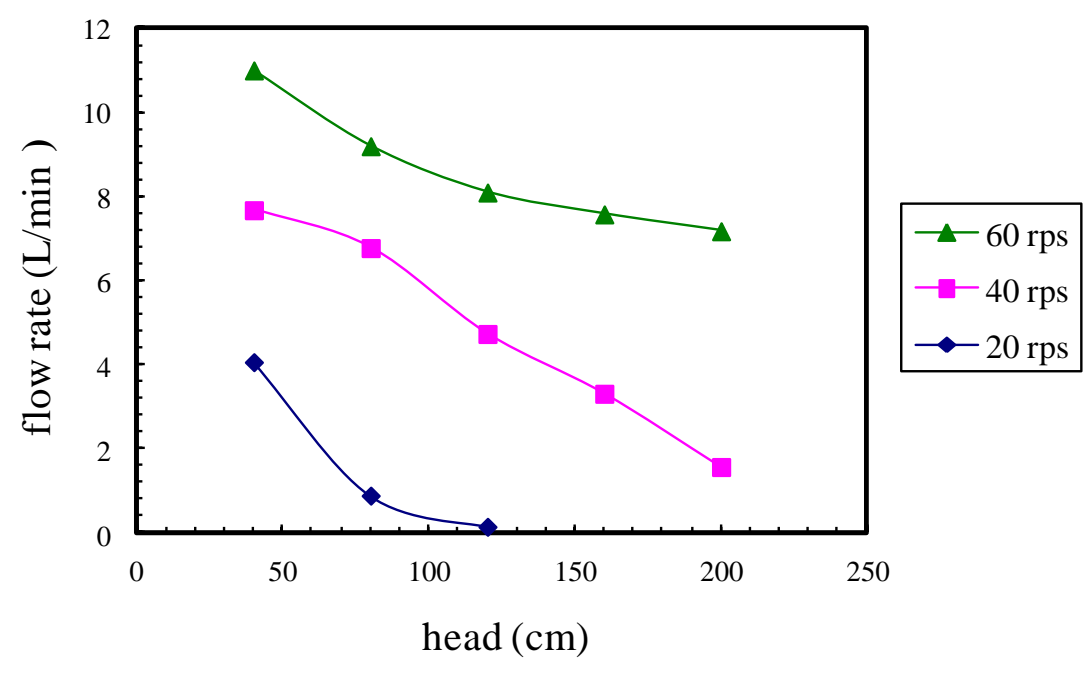

Fig. 2: Liquid nitrogen flow-rate chart. The pump was tested with a $9.5 \mathrm{~mm}$ diameter outlet tube whose open end was maintained at varying heights, and with the shaft rotating at 20, 40, and 60 rps. The head on the $\mathrm{x}$ axis is the height of the outlet relative to the $\mathrm{LN}_{2}$ surface in the Dewar.

\section{Conclusion}

We have designed a compact, centrifugal cryogenic pump for circulating $\mathrm{LN}_{2}$. The combination of an inducer and gas release plate effectively resolved the problems of boiling and cavitation within the pump. The pump can deliver $\mathrm{LN}_{2}$ into a $2 \mathrm{~m}$ head with various flow rates. In four years of small-scale laboratory use the design has proven reliable and cost-effective.

\section{Acknowledge}

The authors would like to thank Sidney Gulick of McGill University, and Daniel Burke and Bruce Zabransky of Argonne National Laboratory, for productive conversations about mechanical engineering and cryogenic pumping. This work was carried out under the auspices of the NSERC, Canada, Application No. 216974, and the U.S. Department of Energy, by Argonne National Laboratory, under Contract No. DE-AC02-06CH11357. 


\section{References:}

[1] Barber-Nichols, (2015). <http://www.bardenbearings.com>.

[2] Flowserve, (2015). <http://www.flowserve.com>.

[3] B.M. Wright, Centrifugal pumping of liquefied gases, J. Sci. Instrum. 44 (1967) 469. doi:10.1088/0950-7671/44/6/417.

[4] T. Haruyama, R. Yoshizaki, A miniature centrifugal pump for an automatic liquid nitrogen filling system, J. Phys. [E]. 19 (1986) 919-921. doi:10.1088/0022-3735/19/11/007.

[5] D. Chopra, H. Babb, Inexpensive liquid nitrogen pump, Rev. Sci. Instrum. 46 (1975) 11261127. doi:10.1063/1.1134371.

[6] M. Abe, T. Takayama, R. Sagiyama, H. Tsuchiya, Y. Torii, M. Nakamura, et al., Development of liquid nitrogen Centrifugal Pump, J. Phys. Conf. Ser. 150 (2009) 012001. doi:10.1088/1742-6596/150/1/012001.

[7] T.L. Merriman, J.W. Kannel, Evaluation of EHD film thickness for cryogenic fluids, Lubr. Eng. 29 (1986) 179-184.

[8] B.N. Zotov, Correlation Between Specific Speed and Cavitation Characteristics of Vane Pumps, Chem. Pet. Eng. 41 (2005) 329-332. doi:10.1007/s10556-005-0112-8.

[9] Barden Corporation, (2015). <http://www.bardenbearings.com/>.

[10] V.S. Lobanoff, R.R. Ross, Centrifugal pumps: design and application, Gulf Pub. Co., 1985.

[11] P.J. Kantert, Manual for Archimedean Screw Pump: Adviser and Decision Guidance for Planners, Owners, Managers and Decision Makers, Hirthammer, 2008.

[12] N.D. Scielzo, G. Li, M.G. Sternberg, G. Savard, P.F. Bertone, F. Buchinger, et al., The $\beta$ decay Paul trap: A radiofrequency-quadrupole ion trap for precision studies, Nucl. Instrum. Methods Phys. Res. Sect. Accel. Spectrometers Detect. Assoc. Equip. 681 (2012) 94-100. doi:10.1016/j.nima.2012.04.035.

[13] G. Li, R. Segel, N.D. Scielzo, P.F. Bertone, F. Buchinger, S. Caldwell, et al., Tensor interaction limit derived from the $\alpha-\beta-v$ correlation in trapped $8 \mathrm{Li}$ ions, Phys. Rev. Lett. 110 (2013) 092502.

[14] J. Van Schelt, D. Lascar, G. Savard, J.A. Clark, P.F. Bertone, S. Caldwell, et al., First Results from the CARIBU Facility: Mass Measurements on the r-Process Path, Phys. Rev. Lett. 111 (2013) 061102. doi:10.1103/PhysRevLett.111.061102. 